\title{
The low down on sphingosine-1-phosphate lyase as a regulator of thymic egress
}

Julie D. Saba*

University of California San Francisco Benioff Children's Hospital Oakland, Children's Hospital Oakland Research Institute, Oakland, USA

\section{Article Info}

\section{Article Notes}

Received: October 13, 2017

Accepted: December 06, 2017

\section{*Correspondence:}

Dr. Julie D. Saba, University of California San Francisco Benioff Children's Hospital Oakland, Children's Hospital Oakland Research Institute, 5700 Martin Luther King Jr. Way, Oakland, CA 94611, USA; Telephone: 510-450-7690; Fax: 510-450-7910, E-mail: jsaba@chori.org

(C) 2017 Saba JD. This article is distributed under the terms of the Creative Commons Attribution 4.0 International License.

\section{Keywords:}

Sphingosine-1-phosphate

Sphingosine-1-phosphate lyase

Thymic egress

Lymphocytes

Trafficking

S1PR

Dendritic cell

Autoimmune disease

FTY720

Fingolimod

\section{ABSTRACT}

After undergoing positive and negative selection in the thymus, surviving mature T cells egress from the thymic parenchyma and enter the bloodstream to participate in adaptive immunity. Thymic egress requires signals mediated by sphingosine-1-phosphate (S1P), a bioactive lipid that serves as the ligand for a family of $\mathrm{G}$ protein-coupled receptors (S1P1-5) expressed on many cell types, including $T$ cells. In the final stage of their development, T cells upregulate S1P1 expression on the cell surface, which enables them to recognize and respond to a chemotactic S1P gradient that lures them into the bloodstream. The gradient is generated by an S1P source close to the site of egress combined with an S1P sink generated by the actions of S1P catabolic enzymes including S1P lyase (SPL), the only enzyme that irreversibly degrades S1P. The requisite contribution of SPL to thymic egress is demonstrated by the profound lymphopenia observed in SPL knockout (KO) mice and wild type mice treated with SPL inhibitors. SPL is robustly expressed in thymic epithelial cells (TECS), which make up the stromal reticular network of the thymus. However, TEC SPL was recently found to be dispensable for thymic egress. In contrast, deletion of SPL in dendritic cells (DCs) - which represent only a small percent of thymic stroma - disrupts the S1P gradient and blocks thymic egress. These recent observations identify DCs as homeostatic regulators of thymic export through the actions of SPL, thereby adding one more piece to the complex puzzle of how S1P signaling contributes to the regulation of T cell trafficking.

\section{Introduction}

After undergoing positive and negative selection in the thymus, mature $\mathrm{T}$ cells egress from the thymic parenchyma and enter the bloodstream as naïve T cells which become a central feature of the adaptive immune system. The migration of mature $\mathrm{T}$ cells from thymus into the circulation, i.e., "thymic output" is generally a constitutive process that steadily adds to the peripheral naïve $\mathrm{T}$ cell pool. However, conditions such as aging, HIV infection, chemotherapy and/or radiation can reduce thymic output and thereby impact immune function ${ }^{1-4}$. The augmentation of thymic output could have therapeutic benefit in some conditions such as to hasten immune reconstitution after bone marrow transplantation, whereas inhibition of thymic output to prevent the release of self-reactive $\mathrm{T}$ cells could be therapeutic in the context of autoimmune disease. A more complete understanding of the physiological mechanisms involved in $\mathrm{T}$ cell egress could lead to novel immunomodulatory strategies for illness and aging.

Sphingosine-1-phosphate (S1P) is a bioactive metabolite generated by the degradation of sphingolipids. S1P produces signals 
that control cell movement, adhesion, survival/apoptosis and differentiation primarily by ligating to a family of five $\mathrm{G}$ protein-coupled receptors (S1PRs) ubiquitously expressed on the surface of mammalian cells. S1P is formed when sphingosine kinases (SphK1 and SphK2) phosphorylate the long chain base sphingosine. Nonspecific lipid phosphate phosphatases (LPPs) and S1P-specific phosphatases (Sgpp1 and Sgpp2) can dephosphorylate S1P, regenerating sphingosine. However, only the intracellular enzyme S1P lyase (SPL) cleaves the S1P carbon backbone and thereby irreversibly degrades S1P to form hexadecenal and ethanolamine phosphate ${ }^{5}$. As such, SPL, encoded by the gene $S G P L 1$, is a critical regulator of cellular, extracellular, tissue and circulating S1P levels. This review will summarize selected insights from the last fifteen years of research regarding the role of S1P signaling and metabolism in T cell egress from the thymus and peripheral lymphoid organs.
Recent findings on the mechanism of SPL's influence on thymic egress and implications regarding modulation of SPL for therapeutic benefit will be highlighted.

\section{T cell development in the thymus}

Bone marrow-derived lymphoid progenitor cells destined to become $\mathrm{T}$ lymphocytes enter the thymus at the cortico-medullary junction (CMJ) and settle in the $\operatorname{cortex}^{6,7}$. There, they upregulate a repertoire of unique and stochastically determined $\mathrm{T}$ cell receptors (TCR) as well as the TCR co-receptors CD4 and CD8 (Figure 1). These "double positive" (DP) thymocytes undergo positive selection, resulting in the removal of $\mathrm{T}$ cells expressing inefficient TCRs $^{8,9}$. Surviving DP thymocytes then differentiate into semi-mature $\mathrm{T}$ cells by upregulating the homing receptor CCR7 and becoming either CD4+ or CD8+ "single positive" (SP) thymocytes. SP thymocytes

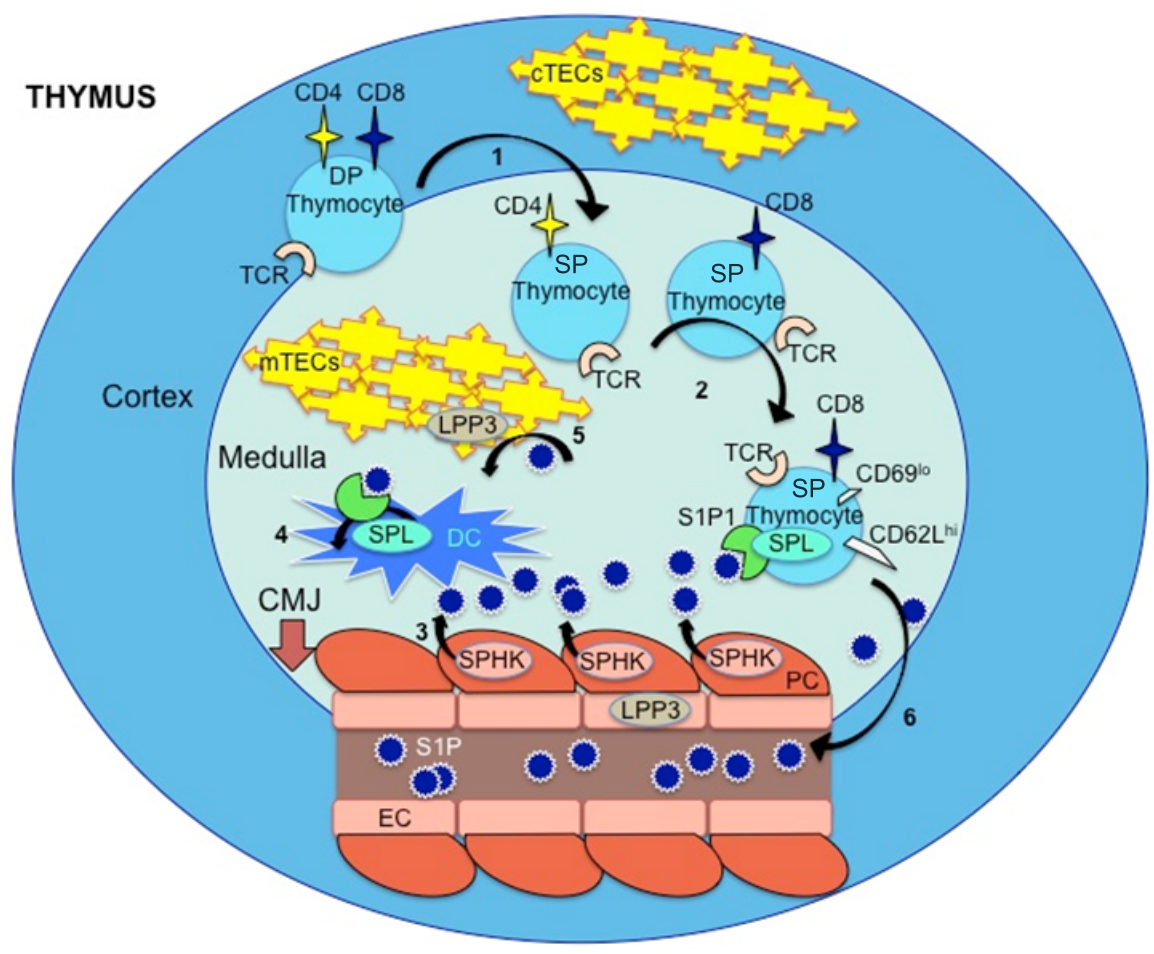

Figure 1. Thymocyte maturation and egress. Thymic cortex is depicted in aqua, thymic medulla in light blue. Cortico-medullary junction (CMJ), the site of T cell egress, is indicated by a dark red bold arrow. Key steps are shown in black arrows. Cortical thymic epithelial cells (cTEC) and medullary thymic epithelial cells (MTEC) that comprise the reticular network of the thymus are depicted in yellow. Bone marrow-derived T lymphocyte precursors emerge from vessels at the CMJ and settle in the thymic cortex, where they begin to express the T cell receptor (TCR) and co-receptors $C D 4$ and CD8 (i.e., double positive cells). 1) $C D 4^{+}$and $C D 8^{+}$double positive (DP) thymocytes migrate from the cortex to the medulla, where they differentiate into $\mathrm{CD} 4^{+}$and $\mathrm{CD} 8^{+}$single-positive (SP) thymocytes. 2) As SP cells mature, they express S1P lyase (SPL) and the transcriptional factor KLF2. The latter leads to increased transcription of s1pr1, resulting in a high expression of surface S1P1 (indicated in bright green). CD62L is also upregulated, and CD69 is downregulated. This process occurs for both CD4+ and CD8+ thymocytes but is depicted here for CD8+ cells for simplicity. 3) Pericytes (PC) that surround blood vessels located at the CMJ harbor sphingosine kinases (SphK) that catalyze formation of S1P (small dark blue circles) and secrete it into the adjacent thymic parenchyma. 4) Thymic dendritic cells (DC) close to the CMJ internalize extracellular S1P by S1PR-mediated uptake, making it accessible to intracellular S1P lyase (SPL). SPL catalyzes the irreversible degradation of S1P. 5) In mTECs and endothelial cells (ECs), S1P is dephosphorylated by LPP3. Both LPP3 and SPL activities are required for egress. 6) The S1P chemotactic gradient produced by the S1P source (pericytes) and S1P sink (DCs and mTECs) interacts with S1P1 on mature thymocytes, thereby facilitating their egress into the bloodstream where high S1P concentrations derived from erythrocytes persist. 
move into the medulla and are subjected to negative selection, which culls lymphocytes with self-recognizing $\mathrm{TCRs}^{8,9}$. Approximately $2 \%$ of $\mathrm{T}$ cells survive both positive and negative selection and subsequently undergo thymic egress.

\section{The initial discoveries linking S1P signaling to thymic egress}

The basic steps involved in thymic egress - and the critical role of S1P signaling in this process - have been illuminated over the past fifteen years through a series of key studies. Historically, both passive and facilitated mechanisms of $\mathrm{T}$ cell egress had been considered, but by 2001 two separate reports provided evidence that $\mathrm{T}$ cell

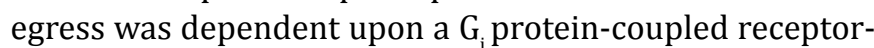
dependent mechanism inhibited by pertussis toxin ${ }^{10,11}$.

In 2002, Mandala and colleagues made a seminal discovery linking S1P and its G protein coupled receptors to the control of thymic egress. They observed that administration of a small molecule called FTY720 to mice could block thymic egress. FTY720 had been developed as a derivative of myriocin, a natural product with immunosuppressive properties shown by its inhibitory activity in the mixed lymphocyte reaction ${ }^{12}$. Myriocin's activity is due to its ability to inhibit sphingolipid biosynthesis $^{12}$. Structure/function analysis of myriocin's immunosuppressive activity led to the development of FTY720 (also known as fingolimod), which was less toxic and exhibited markedly more potent immunosuppressive functions than myriocin at doses below those required to inhibit the mixed lymphocyte reaction ${ }^{13}$. FTY720 pretreatment prevented graft rejection in preclinical models of organ transplantation ${ }^{14}$. Importantly, FTY720 did not induce a globally immunodeficient state, which was a potential advantage over other known immune modulators. Despite these promising findings, its specific mechanism of action was not revealed until Mandala's group discovered that FTY720 prevents T cell egress from thymus and peripheral lymph nodes, and that it does so by targeting S1PRs ${ }^{15,16}$ Treatment of mice with either FTY720 or S1P eliminated T cells from efferent lymphatic vessels, indicating that these interventions blocked egress by trapping lymphocytes at thymic and peripheral lymphoid organ exit sites. Simultaneously, Volker Brinkmann and researchers at Novartis observed similar effects of FTY720 on $\mathrm{T}$ cell trafficking, established that the drug targets S1PRs and - importantly - demonstrated its efficacy in a preclinical model of the autoimmune neurological disorder multiple sclerosis ${ }^{16}$. FTY720 was shown to be a pro-drug that when phosphorylated in vivo by the enzyme sphingosine kinase 2 becomes a structural analog of S1P and targets four out of the five known S1P receptors (all but S1P2) ${ }^{17}$. Using an antigen-challenged mouse model, Rosen's group confirmed that FTY720 prevents the recirculation of naïve $\mathrm{T}$ cells and traps antigen-challenged $\mathrm{T}$ cells in the draining lymph node, but without reducing in vivo or in vitro $\mathrm{T}$ cell proliferation ${ }^{18}$.

\section{The dynamic role of S1P1 in thymic egress}

In 2004, Matloubian et al showed that S1P1 expression by $\mathrm{T}$ and $\mathrm{B}$ cells was critical for their ability to exit lymphoid organs and further demonstrated that S1Pdependent chemotaxis was a dynamic process - namely, that S1P1 was upregulated on the cell surface before thymocytes exited the thymus, and downregulated after lymphocytes underwent stimulation ${ }^{19}$. Downregulation of T lymphocyte S1P1 after antigenic stimulation led to their retention in peripheral lymphoid organs. Similarly, FTY720 induced downregulation of S1P1 on lymphocytes, causing their retention in lymph nodes ${ }^{19}$. Consistent with these findings, Richard Proia's group showed that disruption of the S1PR1 gene in murine $\mathrm{T}$ cells was sufficient to prevent thymic egress ${ }^{20}$. Subsequent studies proved that T-cell intrinsic S1P1 signaling promotes $\mathrm{T}$ cell egress by overcoming retention signals mediated through CCR7 and other $G_{i}$ protein-coupled receptors ${ }^{21}$. The necessity of S1PR-dependent signaling for T cell trafficking was shown to be highly context-dependent by the Bromberg group. They showed that within lymph nodes T cell migration to some signals (i.e., CXCL12) but not to others (i.e., CCL19) required dual chemokine and S1PR stimulation, whereas this was not true of splenic T cells, which did not require S1PR stimulation to migrate to either chemokine ${ }^{22}$.

\section{The contribution of S1P catabolism and transport to thymic egress}

In 2005, Schwab and colleagues demonstrated that S1P1 expression was not the only factor needed to facilitate thymic egress. By reducing local tissue S1P levels, SPL generated a chemotactic gradient that was essential for guiding S1P1expressing mature thymocytes away from thymus and into the blood ${ }^{23}$. The importance of the chemotactic S1P gradient was confirmed by the demonstration that inhibition of SPL activity by tetrahydroxybutylimidazole - a compound found in caramel food coloring known previously to cause lymphopenia ${ }^{24}$ - also blocked thymic egress when administered to mice, consistent with the lymphopenia characteristic of global SPL knockout mice ${ }^{23,25}$. Thus, an S1P chemotactic gradient combined with a receptor system for sensing that gradient are the two fundamental requirements that enable mature naïve $\mathrm{T}$ cells to enter the circulation.

Interestingly, the Schwab group established that the lipid phosphatase LPP3 is also required for thymic egress and that disruption of LPP3 in either Endothelial Cells (EC) or TEC compartments causes a block in thymic egress ${ }^{26}$. Fukuhara and colleagues showed further that S1P export from EC by the S1P transporter Spns2 is also required for egress ${ }^{27}$. Collectively, 
these findings suggest that the S1P gradient is dependent upon multiple activities to produce S1P at the site of egress and eliminate S1P signals elsewhere, thereby shaping the gradient to generate the optimal chemotactic effect.

Additional revelations about the S1P signaling axis and thymic egress

In 2006, Carlson et al demonstrated that mature thymocytes upregulate Kruppel-like factor 2 (KLF2), a transcription factor that promotes the transcription of genes involved in homing and trafficking ${ }^{28}$. S1P1 was found to be a transcriptional target of KLF2 that is upregulated along with CD62L during the final stage of $\mathrm{T}$ cell maturation ${ }^{28}$. Thymocytes lacking KLF2 expression failed to express beta7 integrin, CD62L and S1P1 and were unable to egress the thymus ${ }^{28}$.

Utilizing in vivo labeling of SP CD4+ thymocytes combined with fluorescent microscopy, Zachariah and colleagues showed that mature $\mathrm{T}$ cells exit the thymus through blood vessels located at the $\mathrm{CMJ}^{29}$. They also showed that sphingosine kinase activity within pericytes located at the CMJ produce the critical S1P signal that together with S1P catabolic activities - generates the S1P chemotactic gradient essential for egress.

In 2009, Weber and colleagues, studying SPL knockout mice, established that SPL expression is essential for thymic development and that without SPL toxic ceramides accumulate and new thymic settling is prevented, reducing the availability of thymic early $\mathrm{T}$ cell progenitors ${ }^{30}$. These studies suggest that S1P metabolism plays multiple roles in thymic function and $\mathrm{T}$ cell development in addition to its essential contribution to thymic egress.

Integration of S1P1 signaling within a dynamic immune response system

Similar to their role in thymic egress, S1P gradients and S1P1 signaling are critical regulators of T cell trafficking in the periphery. Lymphocyte migratory responses are highly complex and dynamic, occurring in a tissue-dependent context and responsive to antigen-mediated activation, cytokines associated with the inflammatory state, and regulatory mechanisms that control S1P1 gene expression, protein stability and subcellular localization. Along with numerous other retention and chemotactic signals including CCR7, interferon gamma and others, S1P1 signals are integrated by lymphocytes within a sophisticated and hierarchical response system modulated by environmental cues and conditions. Due to the complexity of this subject and limitations of space, we refer the interested reader to an excellent recent review on this subject ${ }^{31}$.

S1P-targeted drugs: a new class of immune modulators

Although conflicting results over whether FTY720 acts as an S1PR agonist or antagonist created some initial controversy in the field, it is now clear that FTY720 functions as a super-agonist that activates the receptor but also causes its internalization followed by its proteasomal degradation $^{32,33}$. Gatfield and colleagues showed that after the natural S1P ligand causes S1P1 internalization, recycling of the receptor back to the cell surface requires ligand unloading by SPL-dependent S1P catabolism. In contrast, FTY720-P is not catabolized by SPL and remains bound to its target, thereby preventing its recycling ${ }^{34}$. Thus, the ultimate impact of the drug is to provide a chronic blockade of lymphocyte egress caused by the inability of the $\mathrm{T}$ cells to re-express S1P1, thereby protecting target tissues from lymphocyte-mediated inflammation and injury. Importantly, blockade of lymphocyte trafficking by FTY720 was shown to be efficacious in the treatment of chronic relapsing multiple sclerosis ${ }^{35}$. FTY720 and other rationally designed S1P1 targeted drugs are being evaluated in clinical trials for several autoimmune disorders, and early results appear promising ${ }^{36-38}$.

By reducing the S1P gradient, partial inhibition of SPL through pharmacological means also prevents lymphocyte egress from lymph nodes. In preclinical studies, this strategy has been shown to exert protective effects in several $\mathrm{T}$ cell dependent models of inflammation including multiple sclerosis, rheumatoid arthritis, ischemia reperfusion injury, and the genetic disorder Duchenne's muscular dystrophy, which is characterized by a marked inflammatory response within the affected muscles ${ }^{39-43}$.

\section{The cellular source of SPL that generates the S1P chemotactic gradient}

It was assumed by many in the field that thymic epithelial cells (TECs) — which make up the stromal reticular network of the thymus and which express abundant SPL - must harbor the SPL activity that generates the S1P sink needed to strengthen the amplitude of the chemical gradient. To confirm this, we employed Cre/Lox technology to generate transgenic (Tg) mice in which SGPL1 is disrupted selectively in TECs (SPL ${ }^{\mathrm{TEC} \_} \mathrm{KO}$ mice). Interestingly, SPL ${ }^{\mathrm{TEC} \_\mathrm{KO}}$ mice had no egress phenotype ${ }^{44}$. Even more surprisingly, S1P levels in the whole thymus of $\mathrm{SPL}^{\mathrm{TEC}} \mathrm{KO}$ mice were unaffected compared to controls ${ }^{44}$.

Considering that the thymus is composed largely of blood and vascular cell types, we next generated Tg mice in which SPL was disrupted in all hematopoietic cell types and ECs using a similar Cre/Lox strategy. The SPL ${ }^{\mathrm{Mx} 1{ }^{\mathrm{K} 0}}$ mice thus generated exhibited profound accumulation of CD4+, CD8+ and mature $\mathrm{T}$ cells in the thymus, in association with lymphopenia and elevated thymic S1P levels ${ }^{44}$. Extracellular thymic S1P levels at the site of egress were also high, as shown by a complete downregulation of S1P1 on the cell surface of mature thymic $\mathrm{T}_{\text {cells }}{ }^{44}$. Bone marrow 
transplantation studies showed that thymic egress was blocked only when SPL was lacking in the hematopoietic compartment, whereas EC SPL was not essential for thymic egress $^{44}$.

Disruption of SPL in B lymphocytes, monocyte/ macrophages and lymphatic endothelium resulted in no impact on thymic egress ${ }^{44}$. Driving SPL disruption in $\mathrm{T}$ lymphocytes, however, caused a partial block in thymic egress. In addition, S1P in thymic extracellular fluid was higher than normal. Nonetheless, the severity of the egress phenotype was modest compared to that exhibited by $\mathrm{SPL}^{\mathrm{Mx} 1{ }_{1} \mathrm{KO}} \mathrm{mice}^{44}$. This indicated that some other hematopoietic cell type must harbor the SPL pool essential for generating the S1P gradient and sustaining T cell egress.

Dendritic cells (DCs) make up only $0.5 \%$ of thymic cellular content, yet they play important roles in thymic function and $\mathrm{T}$ cell education ${ }^{45}$. DCs are highly efficient antigen-presenting cells and play a seminal role in the development of central tolerance through mechanisms thought to involve the stimulation of immature thymocytes with antigens brought from the periphery by circulating $\mathrm{DCs}^{46,47}$. Thymic DCs are located in the medulla and $\mathrm{CMJ}^{45}$. However, no role for DCs in T cell trafficking had been previously identified. We generated DC-specific SPL knockout mice (SPL ${ }^{\text {DC_KO }}$ mice) lacking SPL in classical and plasmacytoid $\mathrm{DCs}^{48}$. SPL ${ }^{\mathrm{DC} \_\mathrm{KO}}$ mice showed a prominent egress defect, with severe accumulation of SP and mature $\mathrm{T}$ cells in the thymus and reduced $\mathrm{T}$ cells in blood, lymph, lymph nodes and spleen ${ }^{44}$. In addition, their total thymic S1P levels were significantly elevated, and extracellular S1P levels at the site of $\mathrm{T}$ cell egress were substantially increased, recapitulating the finding of SPL ${ }^{\mathrm{Mx} 1 \_\mathrm{KO}}$ mice $^{44}$. Double KO mice lacking SPL in both T cells and DCs exhibited a block in thymic egress that was equivalent in severity to that observed in SPL ${ }^{\mathrm{DC}}{ }_{\mathrm{K} 0}$ mice $^{44}$. Thus, T cell SPL does not add appreciably to DC SPL in regulating thymic egress. To confirm the importance of DC SPL in regulating egress, we performed rescue experiments, taking advantage of a previously reported finding that labeled donor splenic DCs could be traced to the thymus after infusion into recipient mice $^{49}$. The DCs from both SPL wild type and knockout donor mice homed to the recipient thymus in equal proportions after intravenous administration. However, only the infusion of DCs from wild type donors relieved the egress blockade ${ }^{44}$.

Following the uptake of D7-S1P (a stable isoform of $\mathrm{S} 1 \mathrm{P})$ provided in the cell culture medium, we showed that mature bone marrow-derived DCs as well as CD4+ SP thymocytes from SPL flox/flox control mice degraded D7S1P rapidly and did not accumulate S1P, with DCs exhibiting more efficient S1P uptake than thymocytes. In contrast, DCs generated from SPL ${ }^{\mathrm{M} 1 \_K 0}$ mice accumulated S1P over time, demonstrating efficient S1P uptake and dependence upon intracellular SPL for its degradation ${ }^{44}$.

To determine how DCs take up S1P and make it available to SPL, we employed a series of inhibitors to block the three most likely processes involved in S1P uptake, namely S1P1-mediated uptake, receptor-independent endocytosis or dephosphorylation, the latter representing a common mechanism for uptake of phosphorylated drugs and other compounds. Inhibition of S1P1 activity by FTY720 completely blocked S1P uptake into DCs. Inhibition of endocytosis with dynasore and inhibition of LPP ectoenzyme with XY14 reduced S1P uptake to a lesser degree ${ }^{44}$. These findings demonstrate that DCs take up S1P from the extracellular fluid by receptor-mediated endocytosis and degrade it via the actions of SPL (Figure 1).

\section{Conclusions and remaining questions}

Our cumulative results demonstrate that SPL activity contained within thymic DCs is essential for maintaining low extracellular S1P levels at the site of egress, thereby producing the S1P chemotactic gradient essential for enabling mature $\mathrm{T}$ cell entry into the circulation. DCs import S1P via a S1P receptor-dependent mechanism, thereby making it available for degradation by SPL. T cells are also capable of importing S1P and degrading it, but the contribution of T cell SPL to the S1P gradient and thymic egress is nominal compared to that of DCs, based on comparisons of S1P1 cell surface expression on mature thymic T cells, the rates of S1P uptake, and the egress phenotypes of T cell, DC and double knockout SPLdeficient mice. Our findings, in conjunction with the finding by others that vascular and perivascular sources of LPP3, Spns2 and SphK activities are also essential for thymic egress, emphasize the importance of highly localized S1P gradients in regulating immune cell trafficking. Our findings also reveal a novel role for DCs in T cell egress, independent of their other important functions in the development of central tolerance and immune surveillance.

These findings, however, raise many new questions regarding SPL's role in immunity. What purpose does SPL serve in TECs, wherein SPL is so highly expressed and yet not involved in T cell trafficking? What impact does SPL have on $\mathrm{T}$ cell differentiation, the TCR repertoire and the egress of other T cell subsets and NKT cells emerging from the thymus? A complex set of proteins including S1P1, SPL, LPP3, the S1P transporter SPNS2, and sphingosine kinases are all required for proper egress ${ }^{31}$. How do all these players coordinately control S1P metabolism, transport and signaling to maintain efficient egress and shape the S1P gradient? Does DC SPL also maintain localized gradients in peripheral lymphoid organs and within sub-compartments of organs such as spleen, lymph nodes and Peyer's patches? Our study was performed under relatively controlled and static conditions. The immune system, however, is a 
dynamic process that changes during development and aging and that responds to pathogens, drugs and other stimuli. What central or peripheral factors might modulate SPL expression and/or activity in DCs or other cell types and thereby influence lymphocyte trafficking? Considering that $\mathrm{S} 1 \mathrm{P}$ signaling is also important in regulating the trafficking and activation of other immune cell types including DCs, what role does SPL play in the intrinsic functions of innate immune cells?

Recently, we reported the discovery of a novel human syndrome of immunodeficiency, steroid-resistant nephrotic syndrome, adrenal insufficiency, central and peripheral neuropathy, and acanthosis caused by inactivating mutations in $S G P L 1^{50}$. The finding of lymphopenia in the affected patients substantiates the role of SPL in lymphocyte trafficking as elucidated by studies in mice. However, some of the patients in the cohort exhibited other immunological and hematopoietic phenotypes, including abnormalities in natural killer (NK) cells ${ }^{50}$. Combined with another recent report implicating SPL in the regulation of NK cell trafficking and tumor suppression in a cancer metastasis model, it seems likely that SPL plays an important role in NK function that could be harnessed for therapeutic purposes in cancer patients ${ }^{51}$. These findings will require further investigation to elucidate the potential role of SPL in NK cell emergence into the circulation, differentiation, activation and trafficking.

FTY720 is approved by the Food and Drug Administration for the treatment of chronic relapsing multiple sclerosis ${ }^{35}$. Clinical studies testing the utility of FTY720 and more recently developed selective S1P1 antagonists in multiple sclerosis and a range of other autoimmune diseases including inflammatory bowel disease, psoriasis, rheumatoid arthritis are ongoing ${ }^{36}$. Based on positive early results, it seems likely that S1PR targeted drugs represent a powerful new class of immune modulators that will soon make their way to the clinic. Their ability to block lymphocyte-mediated target organ damage without disabling other $\mathrm{T}$ cell functions is a clear advantage over steroids and other immunosuppressive drugs.

Whether SPL-targeted therapy will be useful as an immune modulator remains to be determined. The serious manifestations of human SPL deficiency syndrome raise concerns that sustained and complete pharmacological SPL inhibition for immunomodulation in autoimmune disease, cancer, or to improve thymic output after bone marrow transplantation may produce unacceptable toxicities. On the other hand, temporary, partial or cyclical SPL inhibition to improve the survival of transplanted cells, tissues or organs or to promote immune-mediated cancer cell destruction may have its place in combination with other pharmacological strategies of the future. Additional research will be required to address these possibilities.

S1P signaling and metabolism clearly represent a common mechanistic theme that is reprised in different tissues and cell types of the immune system. As we reveal more about the specific and coordinated functions of S1P and S1P-related proteins in this process and in relation to the dynamic interplay of chemokine signals that influence lymphocyte trafficking, the more it will become possible to harness this system for therapeutic benefit.

\section{Acknowledgments}

This work was supported by CA129438 and funds from the Swim Across America Foundation. We apologize to the many outstanding investigators who have contributed to this field but whom we could not mention due to limitations of space.

\section{Conflict of Interest}

The author has no financial conflicts of interest related to this work.

\section{Abbreviations}

Cortico-medullary junction, CMJ; dendritic cell, DC; double positive, DP; knockout, KO; Kruppel-like factor 2, KLF2; lipid phosphate phosphatase, LPP; natural killer, NK; sphingosine-1-phosphate, S1P; sphingosine-1-phosphate receptor, S1PR; single positive, SP; sphingosine phosphate lyase, SPL; T cell receptor, TCR; thymic epithelial cell, TEC; transgenic, Tg.

\section{References}

1. Jin R, Zhang J, Chen W. Thymic output: influence factors and molecular mechanism. Cell Mol Immunol. 2006; 3(5): 341-50. Epub 2006/11/10. PubMed PMID: 17092431.

2. Douek DC, Betts MR, Hill BJ, et al. Evidence for increased T cell turnover and decreased thymic output in HIV infection. J Immunol. 2001; 167(11): 6663-8. PubMed PMID: 11714838.

3. Palmer DB. The effect of age on thymic function. Frontiers in immunology. 2013; 4: 316. doi: 10.3389/fimmu.2013.00316. PubMed PMID: 24109481; PMCID: 3791471.

4. Sun DP, Wang L, Ding CY, et al. Investigating Factors Associated with Thymic Regeneration after Chemotherapy in Patients with Lymphoma. Frontiers in immunology. 2016; 7: 654. doi: 10.3389/ fimmu.2016.00654. PubMed PMID: 28082988; PMCID: 5186774.

5. Kumar A, Saba JD. Lyase to live by: sphingosine phosphate lyase as a therapeutic target. Expert Opin Ther Targets. 2009; 13(8): 101325. Epub 2009/06/19. doi: 10.1517/14728220903039722. PubMed PMID: 19534571; PMCID: 2774446.

6. Rossi FM, Corbel SY, Merzaban JS, et al. Recruitment of adult thymic progenitors is regulated by P-selectin and its ligand PSGL-1. Nat Immunol. 2005; 6(6): 626-34. doi: 10.1038/ni1203. PubMed PMID: 15880112.

7. Cyster JG. Settling the thymus: immigration requirements. J Exp Med. 2009; 206(4): 731-4. Epub 2009/04/08. doi: jem.20090458 [pii]10.1084/jem.20090458. PubMed PMID: 19349460; PMCID: PMC2715109.

8. Klein L, Kyewski B, Allen PM, et al. Positive and negative selection of 
the T cell repertoire: what thymocytes see (and don't see). Nat Rev Immunol. 2014; 14(6): 377-91. doi: 10.1038/nri3667. PubMed PMID: 24830344; PMCID: 4757912.

9. Takaba $\mathrm{H}$, Takayanagi $\mathrm{H}$. The Mechanisms of $\mathrm{T}$ Cell Selection in the Thymus. Trends Immunol. 2017; 38(11): 805-16. doi: 10.1016/j. it.2017.07.010. PubMed PMID: 28830733.

10. Chaffin KE, Perlmutter RM. A pertussis toxin-sensitive process controls thymocyte emigration. Eur J Immunol. 1991; 21(10): 256573. doi: 10.1002/eji.1830211038. PubMed PMID: 1655469.

11. Lee CK, Kim K, Welniak LA, et al. Thymic emigrants isolated by a new method possess unique phenotypic and functional properties. Blood. 2001; 97(5): 1360-9. PubMed PMID: 11222381.

12. Wadsworth JM, Clarke DJ, McMahon SA, et al. Langridge-Smith PR, Broughton HB, Dunn TM, Naismith JH, Campopiano DJ. The chemical basis of serine palmitoyltransferase inhibition by myriocin. J Am Chem Soc. 2013; 135(38): 14276-85. doi: 10.1021/ja4059876. PubMed PMID: 23957439.

13. Napoli KL. The FTY720 story. Ther Drug Monit. 2000; 22(1): 47-51. PubMed PMID: 10688258.

14. Suzuki S, Enosawa S, Kakefuda T, et al. A novel immunosuppressant FTY720, with a unique mechanism of action, induces long-term graft acceptance in rat and dog allotransplantation. Transplantation. 1996; 61(2): 200-5. PubMed PMID: 8600623.

15. Mandala S, Hajdu R, Bergstrom J, et al. Alteration of lymphocyte trafficking by sphingosine-1-phosphate receptor agonists. Science. 2002; 296(5566): 346-9. Epub 2002/03/30. doi: 10.1126/ science.1070238. PubMed PMID: 11923495.

16. Brinkmann V, Davis MD, Heise CE, et al. The immune modulator FTY720 targets sphingosine 1-phosphate receptors. J Biol Chem. 2002; 277(24): 21453-7. PubMed PMID: 11967257.

17. Kharel Y, Lee S, Snyder AH, et al. Sphingosine kinase 2 is required for modulation of lymphocyte traffic by FTY720. J Biol Chem. 2005; 280(44): 36865-72. PubMed PMID: 16093248.

18. Xie JH, Nomura N, Koprak SL, et al. Sphingosine-1-phosphate receptor agonism impairs the efficiency of the local immune response by altering trafficking of naive and antigen-activated CD4+ T cells. J Immunol. 2003; 170(7): 3662-70. PubMed PMID: 12646631.

19. Matloubian M, Lo CG, Cinamon G, et al. Lymphocyte egress from thymus and peripheral lymphoid organs is dependent on S1P receptor 1. Nature. 2004; 427(6972): 355-60. Epub 2004/01/23. doi: 10.1038/nature02284 nature02284 [pii]. PubMed PMID: 14737169.

20. Allende ML, Dreier JL, Mandala S, et al. Expression of the sphingosine 1-phosphate receptor, S1P1, on T-cells controls thymic emigration. J Biol Chem. 2004; 279(15): 15396-401. Epub 2004/01/21. doi: 10.1074/jbc.M314291200. PubMed PMID: 14732704.

21. Pham TH, Okada T, Matloubian $M$, et al. S1P1 receptor signaling overrides retention mediated by $G$ alpha i-coupled receptors to promote $\mathrm{T}$ cell egress. Immunity. 2008; 28(1): 122-33. Epub 2008/01/01. doi: 10.1016/j.immuni.2007.11.017. PubMed PMID 18164221; PMCID: 2691390.

22. Yopp AC, Ochando JC, Mao M, et al. Sphingosine 1-phosphate receptors regulate chemokine-driven transendothelial migration of lymph node but not splenic T cells. J Immunol. 2005; 175(5): 2913-24. PubMed PMID: 16116177.

23. Schwab S, Pereira J, Matloubian M, et al. Lymphocyte sequestration through S1P lyase inhibition an disruption of S1P gradients. Science. 2005; 309: 1735-9.

24. Houben GF, Penninks AH, Seinen W, et al. Immunotoxic effects of the color additive caramel color III: immune function studies in rats. Fundam Appl Toxicol. 1993; 20(1): 30-7. PubMed PMID: 8432426.
25. Schmahl J, Rizzolo K, Soriano P. The PDGF signaling pathway controls multiple steroid-producing lineages. Genes Dev. 2008; 22(23): 3255 67. Epub 2008/12/06. doi: 22/23/3255 [pii] 10.1101/gad.1723908. PubMed PMID: 19056881; PMCID: 2600765.

26. Bréart B, Ramos-Perez WD, Mendoza A, et al. Lipid phosphate phosphatase 3 enables efficient thymic egress. J Exp Med. 2011; 208(6): 1267-78. Epub 2011/05/18. doi: 10.1084/jem.20102551. PubMed PMID: 21576386; PMCID: 3173249.

27. Fukuhara S, Simmons S, Kawamura S, et al. The sphingosine1-phosphate transporter Spns2 expressed on endothelial cells regulates lymphocyte trafficking in mice. J Clin Invest. 2012; 122(4): 1416-26. Epub 2012/03/13. doi: 10.1172/JCI60746. PubMed PMID: $22406534 ;$ PMCID: 3314466.

28. Carlson CM, Endrizzi BT, Wu J, et al. Kruppel-like factor 2 regulates thymocyte and T-cell migration. Nature. 2006; 442(7100): 299-302. doi: 10.1038/nature04882. PubMed PMID: 16855590.

29. Zachariah MA, Cyster JG. Neural crest-derived pericytes promote egress of mature thymocytes at the corticomedullary junction. Science. 2010; 328(5982): 1129-35. Epub 2010/04/24. doi: science.1188222 [pii] 10.1126/science.1188222. PubMed PMID: 20413455; PMCID: PMC3107339.

30. Weber C, Krueger A, Munk A, et al. Discontinued postnatal thymocyte development in sphingosine 1-phosphate-lyase-deficient mice. J Immunol. 2009; 183(7): 4292-301. Epub 2009/09/15. doi: jimmunol.0901724 [pii]10.4049/jimmunol.0901724. PubMed PMID: 19748984.

31. Baeyens A, Fang V, Chen C, et al. Exit Strategies: S1P Signaling and T Cell Migration. Trends Immunol. 2015; 36(12): 778-87. doi: 10.1016/j. it.2015.10.005. PubMed PMID: 26596799; PMCID: 4832571.

32. Graler MH, Goetzl EJ. The immunosuppressant FTY720 downregulates sphingosine 1-phosphate $G$ protein-coupled receptors. FASEB J. 2004; 18(3): 551-3. PubMed PMID: 14715694.

33. Oo ML, Thangada S, Wu MT, et al. Immunosuppressive and antiangiogenic sphingosine 1-phosphate receptor-1 agonists induce ubiquitinylation and proteasomal degradation of the receptor. J Biol Chem. 2007; 282(12): 9082-9. Epub 2007/01/24. doi: M610318200 [pii] 10.1074/jbc.M610318200. PubMed PMID: 17237497.

34. Gatfield J, Monnier L, Studer R, et al. Sphingosine-1-phosphate (S1P) displays sustained S1P1 receptor agonism and signaling through S1P lyase-dependent receptor recycling. Cell Signal. 2014; 26(7): 157688. Epub 2014/04/08. doi: S0898-6568(14)00131-4 [pii] 10.1016/j. cellsig.2014.03.029. PubMed PMID: 24704119.

35. Kappos L, Antel J, Comi G, et al. Oral fingolimod (FTY720) for relapsing multiple sclerosis. N Engl J Med. 2006; 355: 1124-40. PubMed PMID: 16971719.

36. Chew WS, Wang W, Herr DR. To fingolimod and beyond: The rich pipeline of drug candidates that target S1P signaling. Pharmacol Res. 2016; 113(Pt A): 521-32. doi: 10.1016/j.phrs.2016.09.025. PubMed PMID: 27663260.

37. Scott FL, Clemons B, Brooks J, et al. Ozanimod (RPC1063) is a potent sphingosine-1-phosphate receptor-1 (S1P1) and receptor-5 (S1P5) agonist with autoimmune disease-modifying activity. Br J Pharmacol. 2016; 173(11): 1778-92. doi: 10.1111/bph.13476. PubMed PMID: 26990079; PMCID: PMC4867749.

38. Kunkel GT, Maceyka M, Milstien S, et al. Targeting the sphingosine1-phosphate axis in cancer, inflammation and beyond. Nat Rev Drug Discov. 2013; 12(9): 688-702. Epub 2013/08/21. doi: 10.1038/ nrd4099. PubMed PMID: 23954895; PMCID: 3908769.

39. Weiler S, Braendlin N, Beerli C, et al. Orally active 7-substituted (4-benzylphthalazin-1-yl)-2-methylpiperazin-1-yl]nicotinonitriles as active-site inhibitors of sphingosine 1-phosphate lyase for the treatment of multiple sclerosis. J Med Chem. 2014; 57(12): 507484. Epub 2014/05/09. doi: 10.1021/jm500338n. PubMed PMID: 


\section{4}

40. BagdanoffJT, Donoviel MS, Nouraldeen A, etal. Inhibition of sphingosine 1-phosphate lyase for the treatment of rheumatoid arthritis: discovery of (E)-1-(4-((1R,2S,3R)-1,2,3,4-tetrahydroxybutyl)-1H-imidazol-2-yl) ethanone oxime (LX2931) and (1R,2S,3R)-1-(2-(isoxazol-3-yl)-1Himidazol-4-yl)butane-1,2,3,4-tetraol (LX2932). J Med Chem. 2010 53(24): 8650-62. Epub 2010/11/26. doi: 10.1021/jm101183p. PubMed PMID: 21090716.

41. Bandhuvula P, Honbo N, Wang GY, et al. S1P lyase: a novel therapeutic target for ischemia-reperfusion injury of the heart. Am J Physiol Heart Circ Physiol. 2011; 300(5): H1753-61. Epub 2011/02/22. doi: 10.1152/ajpheart.00946.2010. PubMed PMID: 21335477; PMCID: 3094087.

42. Loh KC, Leong WI, Carlson ME, et al. Sphingosine-1-phosphate enhances satellite cell activation in dystrophic muscles through a S1PR2/STAT3 signaling pathway PLoS One. 2012;7(5):e37218; PMCID: 3351440.

43. Ieronimakis $N$, Pantoja $M$, Hays $A L$, et al. Increased sphingosine-1phosphate improves muscle regeneration in acutely injured mdx mice. Skelet Muscle. 2013; 3(1): 20. Epub 2013/08/07. doi: 2044-5040-320 [pii] 10.1186/2044-5040-3-20. PubMed PMID: 23915702; PMCID: 3750760 .

44. Zamora-Pineda J, Kumar A, Suh J, et al. Dendritic cell sphingosine-1phosphate lyase regulates thymic egress. J Exp Med. 2016; 213(12) 2773-91.
45. Wu L, Shortman K. Heterogeneity of thymic dendritic cells. Semin Immunol. 2005; 17(4): 304-12. Epub 2005/06/11. doi: 10.1016/j. smim.2005.05.001. PubMed PMID: 15946853.

46. Guerder S, Viret C, Luche H, et al. Differential processing of selfantigens by subsets of thymic stromal cells. Curr Opin Immunol. 2012; 24(1): 99-104. Epub 2012/02/03. doi: 10.1016/j.coi.2012.01.008. PubMed PMID: 22296716.

47. Weist BM, Kurd N, Boussier J, et al. Thymic regulatory $\mathrm{T}$ cell niche size is dictated by limiting IL-2 from antigen-bearing dendritic cells and feedback competition. Nat Immunol. 2015; 16(6): 635-41. Epub 2015/05/06. doi: ni.3171 [pii] 10.1038/ni.3171. PubMed PMID: 25939026 ; PMCID: 4439282.

48. Caton ML, Smith-Raska MR, Reizis B. Notch-RBP-J signaling controls the homeostasis of CD8- dendritic cells in the spleen. J Exp Med. 2007; 204(7): 1653-64. Epub 2007/06/27. doi: jem.20062648 [pii] 10.1084/jem.20062648. PubMed PMID: 17591855; PMCID: 2118632.

49. Bonasio R, Scimone ML, Schaerli P, et al. Clonal deletion of thymocytes by circulating dendritic cells homing to the thymus. Nat Immunol. 2006; 7(10): 1092-100. Epub 2006/09/05. doi: 10.1038/ni1385. PubMed PMID: 16951687.

50. Lovric S, Goncalves S, Gee HY, et al. Mutations in sphingosine1-phosphate lyase cause nephrosis with ichthyosis and adrenal insufficiency. J Clin Invest. 2017; 127(3): 912-28. doi: 10.1172/ JCI89626. PubMed PMID: 28165339; PMCID: PMC5330730.

51. van der Weyden L, Arends MJ, Campbell AD, et al. Genome-wide in vivo screen identifies novel host regulators of metastatic colonization. Nature. 2017; 541(7636): 233-6. doi: 10.1038/nature20792. PubMed PMID: 28052056; PMCID: 5603286. 